\title{
Miranda
}

Revue pluridisciplinaire du monde anglophone /

Multidisciplinary peer-reviewed journal on the English-

speaking world

$20 \mid 2020$

Staging American Nights

\section{La question méditerranéenne}

\author{
lain Chambers et Marta Cariello
}

Traducteur : Margherita Orsino

\section{OpenEdition}

Journals

Édition électronique

URL : http://journals.openedition.org/miranda/26487

DOI : 10.4000/miranda.26487

ISSN : 2108-6559

Éditeur

Université Toulouse - Jean Jaurès

Référence électronique

lain Chambers et Marta Cariello, « La question méditerranéenne », Miranda [En ligne], 20 | 2020, mis en ligne le 20 avril 2020, consulté le 16 février 2021. URL : http://journals.openedition.org/miranda/26487 ; DOI : https://doi.org/10.4000/miranda.26487

Ce document a été généré automatiquement le 16 février 2021.

\section{(c) (i) () $\Theta$}

Miranda is licensed under a Creative Commons Attribution-NonCommercial-NoDerivatives 4.0 International License. 


\title{
La question méditerranéenne
}

\author{
lain Chambers et Marta Cariello \\ Traduction : Margherita Orsino
}

1 La question méditerranéenne: on fait ici clairement référence à l'essai inachevé d'Antonio Gramsci qui a été ensuite publié sous le titre La question méridionale. ${ }^{1}$ Dans ce texte, la géographie traçait la configuration du pouvoir. ${ }^{2}$ La subordination du midi au nord de l'Italie et le voisinage de celui-ci avec l'Europe moderne transalpine faisaient et font encore partie d'une cartographie où la Méditerranée et les autres suds du monde sont structurellement inférieurs et subalternes, et ce, dès le début de l'ère moderne. La présumée neutralité scientifique dans la délimitation de l'espace garantit la séparation et la hiérarchie des pouvoirs en présence, selon des lieux définis dans un espace-temps moderne. Les observations de Gramsci sur le pouvoir de la géographie dans la définition d'une spatialité des pouvoirs nous invitent à saisir combien la Méditerranée est un produit culturel et politique et non seulement une donnée géographique ou historique. La Méditerranée émerge historiquement des coordonnées résolument terrestres de la pensée (Eckers 2013). Répondre à la question méditerranéenne signifie donc enregistrer les rapports de pouvoirs qui demandent une narration spécifique par rapport à d'autres réalités historiques et culturelles ${ }^{3}$. Le fait même que ce corps aqueux ait été nommé par ceux qui pensent en être les propriétaires surgit déjà de son nom : pour qui la Méditerranée est la Méditerranée et non le Baḥr al-Rūm (la mer des Grecs) ou bien al-Bahr al-Shāmī (la mer de Syrie)?

2 La stratification des spécificités des différents vecteurs du temps empêche sa réduction à une mesure abstraite et universelle. L'histoire ne saurait être étrangère à ces considérations propres au champ de la physique moderne. L'espace-temps est élastique, sujet à des courbures de forces différenciées composées de distances et de dynamiques. Imposer une règle unique, une narration univoque, reviendrait à remplacer la complexité d'une constellation de procédés ouverts, jamais complètement déterminés, par une cage métaphysique. En termes plus triviaux, cela signifie accepter seulement l'histoire de ceux qui veulent arrêter (et refuser) l'histoire pour permettre que leur point de vue reste le seul qui soit possible et acceptable : « les évènements du 
monde ne se mettent pas en rang comme les Anglais. Ils s'amassent de manière chaotique comme les Italiens » (Rovelli 86).

3 Assemblée matériellement à travers des processus historiques et des pratiques analytiques, la Méditerranée s'est constituée dans la culture européenne selon une combinaison de géographies et de critères. Aujourd'hui, elle est suspendue entre les antiques racines présumées en ruine et les actuels loisirs pour vacanciers, tandis que l'arrivée récente des migrants "illégaux ", accompagnés des ombres de milliers de corps qui gisent sur les fonds marins, a dramatiquement terni cette image et détérioré son histoire. Aujourd'hui, la complexité de la formation historique et culturelle de la Méditerranée revient avec toute sa force. L'arrivée non autorisée du migrant a rouvert cette archive, déchiqueté la géographie qui autrefois l'avait enfermée dans des lieux délimités - ailleurs, de l'autre côté, non en Europe - et a exposé la Méditerranée et l'Europe moderne à une multitude de regards et de voix inattendues. Cette rupture croise aussi d'autres interrogations à plus long terme. Les révoltes récentes contre les régimes autoritaires dans le monde arabe qui ont fait basculer les anciens systèmes et accords, les conflits meurtriers dans les Balkans et la guerre coloniale que l'État d'Israël poursuit contre les Palestiniens depuis 1948 s'ajoutent aux questions qui nous arrivent aujourd'hui d'une nouvelle et inattendue centralité de la Méditerranée remettant en cause non seulement les questions géopolitiques mais également les notions de citoyenneté, de droit et d'appartenance. Nous croyons que c'est justement sur ces confins, avec l'émergence d'autres cartographies, que sont en train de s'effriter les limites du précédent cadrage de matrice quasi exclusivement européenne.

4 La cartographie de notre géopolitique qui pense pouvoir encadrer la formation de cette situation et en expliquer l'évolution semble désormais inappropriée. Ce que ce livre veut montrer, c'est qu'il ne s'agit pas simplement de reconnaître les autres avec leurs histoires et cultures mais de relever les limites de nos dispositifs de connaissance. Plutôt que de rechercher une énième explication académique, détachée et neutre (qui reproduirait inévitablement la "supériorité » universelle de son langage), cherchons à tirer de la Méditerranée, de ses histoires et archives multiples, l'exigence de recevoir une pluralité de situations historiques et culturelles qui nous conduisent vers un processus critique novateur. Sur les traces des perspectives avancées par les études culturelles, postcoloniales et décoloniales, on propose l'adoption d'instruments critiques qui nous permettent de réorienter la question de la Méditerranée vers de nouveaux registres. Cette nouvelle cartographie avec une nouvelle narration de la Méditerranée évoque naturellement les géographies plastiques de la déterritorialisation et de la reterritorialisation : un déracinement et une réorientation des interprétations usuelles.

5 Poser la question de qui, quoi, comment et pourquoi cadre et explique la Méditerranée, amène à une évaluation critique de l'actuelle économie politique des savoirs (et du pouvoir). Écouter les langages employés pour raconter la Méditerranée et traverser les espaces où ces langages sont transmis et traduits signifie plier la carte que nous avons héritée, sans l'effacer, pour créer ainsi une profondeur historique et critique qui redessine les lignes d'une Méditerranée différente, encore à venir. 


\section{Penser avec le plongeur}

6 Penser avec le plongeur. Un corps masculin, noir de peau, défie la version européenne du Christ, de la Vierge et des héros grecs, tous blancs de peau et ariens ; un corps qui, il y a deux mille cinq cents ans, plonge avec grâce à travers l'air, regardant de ses yeux grand ouverts le futur. Cette fameuse peinture se trouve sous le couvercle d'un sarcophage (la Tombe du Plongeur) et était destinée à rester invisible. Le tombeau a été retrouvé et ouvert il y a cinquante ans et, devenu visible, il illumine notre présent d'une reconfiguration du passé. Cette figure souple, en plein vol, est entourée, sur les quatre parois intérieures, de figures masculines assises en un symposium. Ce sarcophage appartient à la civilisation grecque de Posidonie, mieux connue sous le nom de Paestum, sur la côte Tyrrhénienne au sud de Salerne, près de l'estuaire du fleuve Sele. En tant que colonie grecque, Paestum faisait partie des cités-États du Péloponnèse qui s'étendaient en Asie Mineure sur la «mer obscure de vin» d'Homère, vers les steppes donnant sur la mer Noire au nord et à l'ouest, en Italie du sud et en Sicile, jusqu'aux côtes de l'Espagne et de la France actuelles. Comme dans tous les colonialismes, les populations indigènes furent conquises, soumises et rendues esclaves. La terre n'était pas vide. Le contrôle devait être arraché aux autorités locales, du sang devait être versé, des vies ôtées (Zuchtriegel, Carter 2015). Cela signifia l'acquisition brutale du sol de la part de quelqu'un, l'imposition de sa gestion politique, d'une culture et d'une mémoire importées sur le territoire. Aujourd'hui une grande partie de ces «détails» ont disparu, perdus dans les mythes d'une nostalgie européenne de la pureté et de la noblesse présumées de ses origines. Pourtant le rappel constant aux origines classiques dans les empires plus récents est affiché dans les motifs architecturaux de toutes les capitales occidentales modernes - de Londres à Paris, du Berlin impérial à Washington et à la Rome fasciste - où l'architecture propose une blancheur des édifices classiques alors que dans l'antiquité ils étaient peints et bariolés.

7 Voilà ce qui revient à penser l'analyse historiographique en termes d'opération anachronique. Ouvrir le tombeau, ouvrir l'archive, dessiner rapidement - comme ces peintres anonymes qui n'ont eu que quelques heures pour terminer leur œuvre avant que l'on ne ferme le couvercle, en principe, à jamais - revient à suggérer un ensemble de connexions et de coordonnées avec lesquelles nous pourrions choisir de naviguer à travers la matrice afro-asiatique-européenne de la Méditerranée. Cela revient aussi, sans abandonner les compétences disciplinaires qui ont permis de mettre au jour ce passé, à refuser la réduction de ces matériaux à un unique inventaire du temps.

8 Nous devons adopter une relation plus ironique avec les origines, établir une archéologie de l'archéologie, découvrir une autre généalogie qui ne soit pas le seul reflet de la quête européenne du pouvoir. Atteignant le cœur de la «civilisation européenne ", ses origines grecques et méditerranéennes, d'autres questions sont soulevées, d'autres géographies qui mènent à une autre compréhension et à d'autres axes d'interprétation qui rendent ce passé, apparemment lointain, proche et déferlant en puissance. La plate taxinomie du temps, où tout est à sa place chronologiquement et culturellement, est ainsi soudainement interrompue et fractionnée, prête à composer un autre collage avec d'autres significations. Comme la peinture du plongeur, exécutée pour des yeux non voyants, maintenant redécouverte et exposée, nous pouvons nous aussi prendre en considération des éléments cachés et sédimentaires qui proposent 
d'autres souvenirs. Il y a aussi la question de la propriété : qui a le droit de raconter et pourquoi ? Selon quel type de généalogie la mémoire est-elle acquise et autorisée ? Essayer de répondre à ces questions mène à proposer un déplacement des principes sous-jacents aux sciences humaines et sociales et de leurs normes et législations.

Briser l'impératif philologique et réassembler ces éléments dans une configuration autre nous invite à assumer une profonde responsabilité concernant notre langage ; nous reconnaissons notre précarité et notre vulnérabilité constantes face à un passé que nous n'allons jamais récupérer ou posséder complètement. Ce passé est encore reconnu et recomposé, collectionné et enregistré, il trace et modélise notre futur. Cela signifie rendre aux objets toute l'épaisseur de leur lignage culturel et la mémoire historique qui résonne en eux, dans une archive en connexion avec leur futur possible. Le but est celui de restituer l'histoire elle-même à une autre histoire et de couper le lien automatique avec l'idée de détachement scientifique en tant que garantie de notre langue et connaissance, en assumant la responsabilité du langage et de la mémoire.

En reconnaissant dans la colonisation grecque en Méditerranée non seulement un empire ou une thalassocratie mais aussi le flux migratoire depuis les villes grecques avec les exils et les diasporas par lesquels a commencé l'entreprise coloniale, on ouvre une brèche dans le temps qui rapproche ce passé des questions contemporaines. Établir un comptoir, pratiquer la colonisation, organiser le territoire d'après un ordre culturel donné, expérimenter, contester et absorber l'hybridation étaient les éléments fondamentaux de l'expérience de Paestum environ deux mille cinq cents ans en arrière et restent valables encore aujourd'hui. Dans cette perspective, on peut établir un archipel qui n'est pas limité à l'espace géographique mais qui se situe dans le temps et s'offre à son exploration.

11 Dans les singularités que nous rencontrons nous pouvons saisir les traces d'une constellation commune qui rend le passé intelligible et permet de comprendre les projections à venir. Si le plongeur dans la tombe est la preuve d'une culture migratoire et hybride - celle de l'Italie méridionale où la civilisation grecque rencontre les cultures étrusque, romaine et lucanienne - il indique également une Méditerranée migrante, aux directions multiples, qui a porté plusieurs noms : phénicienne, grecque, carthaginoise, romaine, byzantine, arabe, normande, génoise, catalane, vénitienne, ottomane... Penser ces noms et histoires signifie encore une fois rouvrir l'archive et insister sur une fluidité qui déborde au-delà des confins terrestres de ce qui aujourd'hui correspond principalement à une narration nationale de cette géohistoire complexe ; c'est ouvrir dans le symposium d'aujourd'hui, enivré par les alcools du déterminisme néolibéral, des débats plus profonds qui transforment la question de la migration moderne, et la valeur périphérique qu'on lui attribue en termes socio-économiques, en phénomène central, en moteur des cultures méditerranéennes et vecteur de la modernité.

12 Les questions d'appartenance traversent nos confins juridiques, culturels, historiques et restent sans réponse. L'accès à la citoyenneté, le droit à la narration, le droit d'avoir des droits dérangent l'ordre politique en place (Arendt). Le migrant devient un chiffre, une non-personne, dont la pratique et la présence décodifient les relations asymétriques du pouvoir qui orchestrent la violence arbitraire du présent (Dal Lago). Cette approche émane d'une perspective critique selon laquelle l'espace est histoire (Carter 1996). Cela ne signifie pas simplement que l'histoire se vérifie en un lieu précis, mais plutôt que le lieu lui attribue une forme et une substance. On déplace ici 
l'attention à la chronologie des évènements et aux échelles de la temporalité vers l'échelle de son écologie matérielle car, pour le dire avec Franco Farinelli, la géographie est histoire (Farinelli). Ainsi la Méditerranée se dérobe au cadrage statique et au panorama des narrations historiques officielles pour se faire, à son tour, histoire. En pensant à, et avec, la complexité historique et culturelle de la formation de la Méditerranée, cherchant à la définir et à la configurer, on s'aperçoit qu'il est nécessaire de dépasser les frontières nationales, les confins des champs du savoir (suivant les défis des études culturelles et postcoloniales) mais aussi de désorienter et de réorienter les coordonnées épistémologiques habituelles.

Dans ce livre, l'étude de la Méditerranée devient l'occasion d'expérimenter une série de propos qui défont la représentation d'une raison capable de rendre le monde transparent à sa propre volonté. Insister sur la valeur historique et politique des formes de vie et de culture qui échappent à la cage rationnelle où tout est réduit à une grammaire de la pensée unique, équivaut à proposer une autre Méditerranée et un autre mode de penser et de pratiquer le monde. Ici, les arts visuels, musicaux et littéraires ont beaucoup à nous apprendre. Ils nous fournissent des langages différents avec lesquels composer une autre cartographie et faire émerger une Méditerranée qui ne rentre plus dans la définition à sens unique, confinée dans une logique académique et disciplinaire, mais qui s'offre à d'autres ordres critiques inattendus et novateurs (Chambers, Ianniciello). En même temps, on démonte la dialectique usuelle du progrès historique, non pour effacer l'histoire mais pour interrompre sa linéarité abstraite et la reconfigurer d'après une stratification matérielle et temporelle qui s'effectue dans le présent. La Méditerranée devient à la fois un laboratoire de la modernité du point de vue historique et culturel et un instrument de provocation épistémologique nous permettant d'en réinterpréter le sens dans le cadre de la modernité.

visions et les définitions de la Méditerranée dont nous avons hérité - avec leurs limites culturelles et linguistiques, l'on adopte un certain scepticisme critique face à la construction coloniale et à l'invention historique de la Méditerranée, largement soutenue par le mythe de l'ordre impérial romain (Tanzarella 18-20). La question de la définition de ses frontières historiques, politiques et théoriques devient presque toujours une question exclusivement européenne. En un mot, l'espace géographiquement défini comme " Méditerranée " ne coïncide pas avec une unité historique et culturelle: il est surdéterminé dans une perspective européenne. Nous pensons que de la friction entre une spatialité partagée et des histoires différenciées peut surgir une problématique que nous appellerons simplement "La Méditerranée " sans nous imposer la nécessité immédiate de la définir, la laissant décanter, en tant que questionnement et horizon à la fois.

15 Évidemment, compte tenu des rapports de pouvoir actuels, la Méditerranée comme définition et pratique politique appartient à l'ordre moderne européen et occidental qui, à son tour, est le calque d'un ordre colonial. Mais nous enregistrons aussi les histoires et cultures subalternes et subordonnées qui l'ont traversée et qui constituent le côté obscur et refoulé de la spécificité spatio-temporelle de cette constellation géohistorique et critique.

16 Nous essayons, ici, de raisonner sur un sujet sans nécessairement le rationaliser. Cette tentative critique se reflète dans le style délibérément épisodique, quasi par fragments, tesselles d'un discours à venir. 

forme de l'essai critique et celle de l'article scientifique (Adorno). L'essai critique dont Walter Benjamin et Hannah Arendt figurent parmi les plus grands auteurs propose un mode d'écriture et de pensée qui s'oppose à une vérité garantie par les protocoles disciplinaires. Ainsi on éradique l'habitude de faire appel à la sacro-sainte véridiction garantie par le caractère scientifique de l'écrit et l'idéal absolu d'une raison qui ferait coïncider la rationalité avec son objectivité présumée. Dans ce genre de récit scientifique, les divagations et les réflexions constituent une hérésie, puisqu'elles sont comme des pauses, des intervalles qui annuleraient la rationalité discursive. C'est pourquoi l'on recherche la méthode dans le processus de la compréhension de soi, tout en réfutant l'illusion d'une quelconque activité historique en dehors de l'histoire. La distinction élaborée par Adorno acquiert chaque jour plus de sens, alors même que la machine académique s'aligne davantage avec ce qu'on appelle la gestion transparente du marché, en substituant les consciences critiques par les compétences techniques.

Contre ce mécanisme, nous jugeons nécessaire, comme le disait Adorno, d'insister sur tout ce qui " pourrait irriter ou être dangereux », pour découvrir la mémoire d'une connaissance non conceptuelle qui adhère à la pensée (Adorno). L'algèbre du pouvoir qui produit connaissance et histoire universelles alors qu'elle discrédite d'autres connaissances locales et indigènes, donc limitées, correspond aux pouvoirs qui forment et ordonnent le monde de manière à faire coïncider leurs coordonnées conceptuelles et matérielles. Tel que l'agenda néolibéral le propose, il n'y a pas d'alternative. Cependant si nous acceptons de remettre en question notre cage conceptuelle, nous devons alors reconnaître que l'économie de la connaissance actuelle se fonde sur une distribution précise, et précaire, des pouvoirs. La prétendue universalité de cette disposition n'efface en rien la spécificité de sa formation historique et de sa situation culturelle à l'intérieur d'une économie politique qui colonise le monde, en s'imposant comme ordre universel.

19 C'est en longeant cette faille que nous désirons bâtir nos analyses et creuser nos parcours critiques.

\section{BIBLIOGRAPHIE}

Adorno, T. W. Il saggio come forma. In Note per la letteratura. Turin: Einaudi, 2012.

Arendt, H. Le origini del totalitarismo. Turin: Einaudi, 2009.

Carter, P. Metabolism. The Exhibition of the Unseen. Victoria: Lyon Housemuseum, 2015.

Carter, P. The Lie of the Land. Londres : Faber \& Faber, 1996.

Chambers, I. Mediterraneo Blues. Musiche, malinconia postcoloniale, pensieri marittimi. Turin: Bollati Boringhieri, 2012.

Chambers, Iain, et Cariello, Marta. La questione mediterranea. Milan: Mondadori, 2019.

Dal Lago, A. Non-persone. L'esclusione dei migranti in una società globale. Milan: Feltrinelli, 2005. 
Ekers, M. et al. Gramsci. Space, Nature, Politics. Chichester: Wiley-Blackwell, 2013.

Farinelli, F. Geografia. Un'introduzione ai modelli del mondo. Turin: Einaudi, 2003.

Gramsci, A. La questione meridionale. Createspace Independent Pub., 2012.

Ianniciello, C. Migrations, Arts and Postcoloniality in the Mediterranean. Londres : Routledge, 2018.

Rovelli, C. L'ordine del tempo. Milan : Adelphi, 2017.

Tanzarella, G. Perspectives et stratégies pour la Méditerranée. Arles : Fondation Sud, 1998.

Zolo, Danilo. « La questione mediterranea ». In L'alternativa mediterranea. Ed. Cassano, F., Zolo, D. Milan: Feltrinelli, 2007.

Zuchtriegel, G. Colonization and Subalternity in Classical Greece : Experience of the Nonelite Population. Cambridge: Cambridge University Press, 2017.

\section{NOTES}

1. Texte disponible: Gramsci, A. La questione meridionale. Createspace Independent Pub., 2012. Le titre d'origine est : Alcuni temi della quistione [sic]meridionale publié à Paris en 1930.

2. Un thème relancé par Danilo Zolo avec son essai : «La questione mediterranea». In L'alternativa mediterranea. Ed. Cassano, F., Zolo, D. Milan : Feltrinelli, 2007.

3. On a simplifié la transcription.

\section{INDEX}

Thèmes : Arts of the Commonwealth

\section{AUTEURS}

\section{IAIN CHAMBERS}

Professeur des universités

Università Orientale di Napoli

\section{MARTA CARIELLO}

Maître de conférences

Università della Campania 